ELECTRONIC LETTER

\title{
Recurrent deletion of a region containing exon 24 of the $R B 1$ gene caused by non-homologous recombination between a LINE-1HS and MER21B element
}

\author{
P Albrecht, J Bode, K Buiting, A K Prashanth, D R Lohmann
}

J Med Genet 2004;41:e122 (http://www.jmedgenet.com/cgi/content/full/41/12/e122). doi: 10.1136/jmg.2004.021923

A bout $10 \%$ of the mutations that are known to cause human monogenic disease are deletions of more than 20 bp (gross deletions). To date, 2127 disease causing gross deletions have been listed in the Human Gene Mutation Database (www.hgmd.org). ${ }^{1}$ For only few of these mutations, breakpoints have been determined at the sequence level because this information is often of little relevance in clinical diagnostics. Those gross deletions that have been analysed in greater detail most often show DNA sequences with direct or inverted similarity at $5^{\prime}$ and $3^{\prime}$ breakpoints. The presence of repeated sequences has suggested models that help to explain the formation of these mutations. ${ }^{2}$

Mutations in the $R B I$ gene can cause retinoblastoma, a childhood tumour of the eye. We have searched for gross deletions in samples of constitutional and tumour DNA from patients with this tumour. Most of these samples had previously been screened for $R B 1$ gene point mutations but with negative results. Using quantitative multiplex polymerase chain reaction $(\mathrm{PCR})^{3}$ we identified a spectrum of gross deletions that was heterogeneous with respect to extent and location. In three patients, we found deletions in a region that contains exon 24 of the $R B I$ gene. Further analysis showed that the $5^{\prime}$ and $3^{\prime}$ deletion breakpoints of these three mutations are located close to each other in an LiHS and MER21B element, respectively. ${ }^{4}$ These two DNA elements belong to different classes of interspersed repetitive DNA. The regions surrounding the $5^{\prime}$ and $3^{\prime}$ breakpoints do not show any sequence similarity. However, they are localised at the borders of strong scaffold/matrix attachment elements that mark the position of recombinogenic DNA structures.

\section{METHODS \\ Samples}

DNA from peripheral blood and from fresh frozen retinoblastoma samples was extracted as described previously. ${ }^{5}$ We investigated constitutional DNA from 60 patients with bilateral or familial retinoblastoma and tumour DNA from 85 patients with isolated unilateral retinoblastoma. Most of these samples had previously been searched for point mutations by Single-strand conformation polymorphism, heteroduplex analysis, or denaturing high performance liquid chromatography. ${ }^{67}$ DNA from tumour samples had also been analysed for hypermethylation of the $\mathrm{CpG}$ island associated with the promoter of the $R B 1$ gene $^{8}$ and for loss of constitutional heterozygosity (LOH) at STR loci RBi2 and RB1.20.

\section{Quantitative multiplex PCR}

Quantitave multiplex PCR was performed using the multiplex PCR assay described by Richter et $a l^{3}$ with only a few minor adaptations of primer sequences and controls. Products were analysed on an ABI 3100 Genetic Analyzer

\section{Key points}

- Retinoblastoma, a malignant tumour of the eye, is caused by mutations in the RB1 gene. About 15-20\% of oncogenic mutations in this gene are gross deletions.

- We have identified a recurrent deletion of a region containing exon 24 of the $R B 1$ gene in peripheral blood of three patients with retinoblastoma. The $5^{\prime}$ and 3 ' deletion breakpoints of these deletions are clustered in an L1HS and MER21B element, respectively.

- The region of the deletion breakpoints does not show DNA sequences with direct or inverted similarity. However, the breakpoints are localised at the borders of strong scaffold/matrix attachment elements that mark the position of recombinogenic DNA structures.

- Our findings emphasise the role of scaffold/matrix attachment regions for deletion formation in humans.

(Applera). Peak integrals were determined using GeneScan and Genotyper software (Applera).

\section{Long range $P C R$ and sequencing}

Long range PCR of a fragment spanning intron 23 to intron 26 of the RBI gene was performed as described by Bremner et al. ${ }^{9}$ To obtain smaller fragments spanning the deleted region, two long range PCRs were performed with primers GCT GGT TCA ATA TAC CA AAT CAA TA ATG (RBgl66248se), TCA TCT GAT CCT TCA ATA TCA AAG CGT AGT (RBg 173837as), and AAA AAT ATA AGC TCT CAG GGG AAT GGA GAT (RBg171908as) (fig 1). To detect both normal and mutant alleles in heterozygous samples, an additional long range PCR was performed using three primers in one reaction: RBgl66248se, RBgl71908as, and AGT TCA GAA TGA TGT ATT TAT GCT CAT CTC TGC (RBgl70286se) (fig 2). $\mathrm{RBg} 170286 \mathrm{se}$ binds to a sequence that is deleted in all three mutant alleles investigated here (fig 3A). PCRs with these three primers on samples heterozygous for one of the exon 24 deletions identified result in a product specific for the deleted allele and in a $1.6 \mathrm{~kb}$ product from the normal allele. For long range PCRs we used the Roche Expand Long Template kit (Roche, Mannheim, Germany).

For sequencing, the PCR products obtained with primers RBgl66248se and RBgl71908as were separated on a $1 \%$ agarose gel, cut out, and eluted. As sequencing primers we

Abbreviations: LINE, long interspersed nuclear elements; $\mathrm{LOH}$, loss of constitutional heterozygosity; PCR, polymerase chain reaction; SIDD, stress induced duplex destabilisation; S/MARs, scaffold/matrix attachment regions 


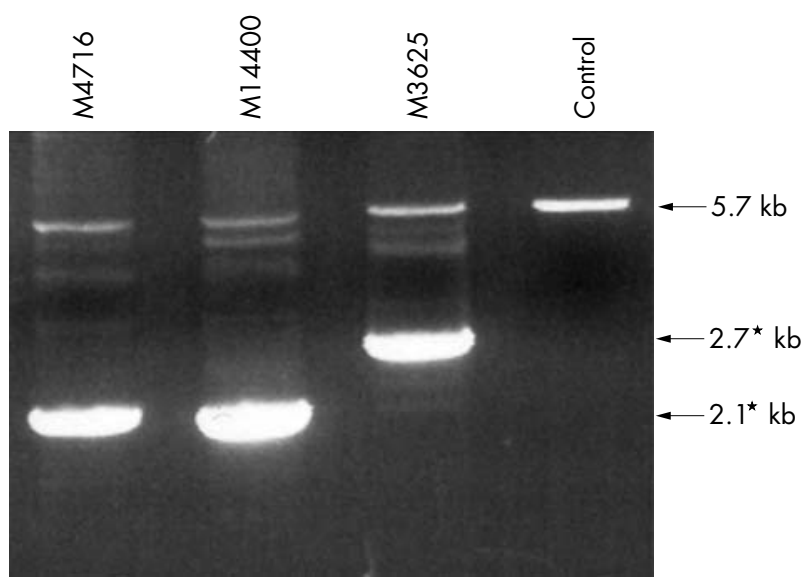

Figure 1 Long range PCR with primers RBg166248se and $\mathrm{RBg} 171908$ as (for primer localisation also see fig 3A). PCR from DNA from a normal control shows the expected $5.7 \mathrm{~kb}$ product only. All three patients (blood DNA from M4716, and tumour DNA from M1 4400 and M3625) also show smaller junction products (labelled with asterisks) from $R B 1$ alleles with deletion mutations.

used RBgl66248se, RBgl71908as, AGG ACA CAA ACA AAT GGA AG (RBgl66914se), and AAT TTA GGA TGG AAG CTG GT (RBgl71317as). The products of sequencing reactions were analysed on an ABI 3100 Genetic Analyzer. The results of sequence analysis were compared using Lasergene sequence analysis software (DNASTAR, Madison WI, USA).

\section{RESULTS AND DISCUSSION}

Gross deletions were identified in 41 patients with retinoblastoma. In line with the results reported by Richter et $\mathrm{al}^{3}$ and Houdayer et $a l^{10}$ the spectrum of gross deletions was heterogeneous with respect to extent and localisation. In both our data set and the data sets published by Richter et al and Houdayer et al, recurrent deletions that affect only a single exon are infrequent. Richter et al identified two mutations of this kind: a deletion of exon 17 in three patients and of exon 13 in two patients; Houdayer et al identified deletions of exon 2 and 8 in one patient each and a deletion of exon 3 in two patients. The breakpoint sequences of these deletions were not reported in the study of either group. In our series, three samples (peripheral blood DNA from patient M4716, tumour DNA from patient M14400, and tumour DNA from patient M3625) showed a deletion of exon 24 in quantitative multiplex PCR. All three samples had been screened for point mutations. The tumour samples from patient M14400 and from patient M3625, which showed no LOH at intragenic STR loci, were found to be heterozygous for a g.162237C $\rightarrow$ T $($ R787X) and a g.76999A $\rightarrow$ G (IVS15-2A $\rightarrow$ G) transition, respectively. To confirm and further characterise the gross deletions identified in the three samples, we performed long range PCR. Agarose gel electrophoresis of products spanning intron 23 to intron 26 and intron 23 to intron 24 showed smaller than expected DNA bands in all three samples (fig 1). Because of preferential amplification of the smaller products from mutant alleles, there was poor amplification of the products specific for the normal allele. We designed a three-primer PCR to obtain a more balanced product ratio in a single PCR assay. The result of the threeprimer PCR indicated that, in constitutional DNA, all three patients are heterozygous for the mutant allele (fig 2). In patient M3625 this finding was unexpected because he had isolated unilateral retinoblastoma and less than $15 \%$ of these patients are carriers of $R B 1$ gene mutations. ${ }^{37}$ All three deletions occurred de novo because analysis of peripheral blood DNA from parents showed only normal $R B 1$ alleles. On the level of the transcript the most probable consequence of all three gross deletions is loss of the $31 \mathrm{bp}$ that correspond to exon 24 , which results in a shift of the reading frame with premature termination eight triplets downstream. Splice donor site mutations in intron 24 that result in skipping of exon 24 lead to identical mutant transcripts. Mutations that involve this site have been reported in patients with bilateral and familial retinoblastoma. ${ }^{11}$ Therefore, the gross deletions involving exon 24 identified here most likely cause predisposition to retinoblastoma in these patients. We further analysed the products of long range PCR by direct sequencing. Sequencing results were compared to the published sequence of the RBI gene (GenBank accession number L11910) and to the results of sequence analysis of a sample without deletion. Patient Ml4400 showed a deletion of 3605 bp (fig 3). The $5^{\prime}$ breakpoint is located in intron 23 at nucleotide 167157 ( $\mathrm{RBg} 167157)$ in a region of $6017 \mathrm{bp}$ with high sequence similarity to an L1HS, a member of the family of long interspersed nuclear elements (LINE). ${ }^{12}$ The $3^{\prime}$ breakpoint of the mutation is located in intron 24 at RBgl70762 within a $764 \mathrm{bp}$ region with similarity to the DNA transposon MER21B. Interestingly, the $5^{\prime}$ and $3^{\prime}$ breakpoints of the deletions identified in the other two patients are located in the same interspersed repetitive DNA elements. In patient M4716, the $5^{\prime}$ and $3^{\prime}$ breakpoints are located after nucleotide position RBgl67672 and RBg171215, respectively. In addition to loss of bases $\mathrm{RBgl} 67673$ to 171214, the mutant allele also shows an insertion of $19 \mathrm{bp}$. A BLASTn sequence comparison of this inserted sequence to the human genome showed no significant similarities to any sequence mapped on chromosome 13. The deletion identified in the sample of patient M3625 is more complex. The mutant allele may be described as a combination of a $2988 \mathrm{bp}$ deletion (dell67988^170975) and an insertion of $3 \mathrm{bp}$ (ins 167988insACT). However, this interpretation cannot account for four mismatches located $95 \mathrm{bp}$ (RBg 167893), $68 \mathrm{bp} \quad(\mathrm{RBg} 167920), \quad 51 \mathrm{bp} \quad(\mathrm{RBg} 16737)$, and $1 \mathrm{bp}$ (RBgl67988) upstream of the most distal possible 5' breakpoint location. A BLASTn similarity search against the human genome showed that the $95 \mathrm{bp}$ sequence ranging from the first mismatch ( $\mathrm{RBg} 167893)$ to the $5^{\prime}$ breakpoint $(\mathrm{RBgl67988})$ is identical only to one sequence (in the opposite direction) that is located in intron 17 of the $R B I$ gene (RBg79218 to RBg 79123) (fig 3). This region in intron 17 is situated in a $1.3 \mathrm{~kb}$ long L1PA5 element ( $\mathrm{RBg} 78540$ to RBg79873), a truncated member of the LINE family.

Recurrent loss of only a single exon of the $R B I$ gene is rare. Intriguingly, there is a clustering of the locations of $3^{\prime}$ and $5^{\prime}$ deletion breakpoints of the exon 24 deletions identified here. The $5^{\prime}$ breakpoints have a maximum distance of $830 \mathrm{bp}$ and are located in an LIHS LINE. This L1HS has 97\% similarity to the full length retrotransposition competent Ll (L1.3, GenBank accession no. L19092) but has acquired

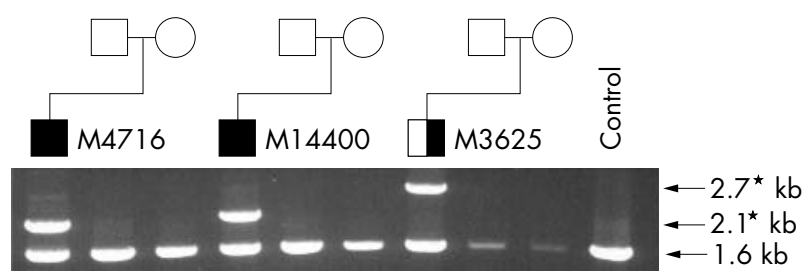

Figure 2 Three-primer PCR with the same primers as in fig 1 plus RBg170286se (see also fig 3A). The presence of the wild type allele is indicated by a PCR product of $1.6 \mathrm{~kb}$. PCR products from RB 1 alleles with deletion mutations (labelled with asterisks) are only detected in the patients (blood DNA from M4716, M14400, and M3625). Due to preferential amplification of the shorter fragments, the $5.7 \mathrm{~kb}$ fragment is not detected on the gel. 
A
L1PA5
L1HS
MER21B

Exon 17

23

24

25

$|+|$

$70 \mathrm{~kb}$
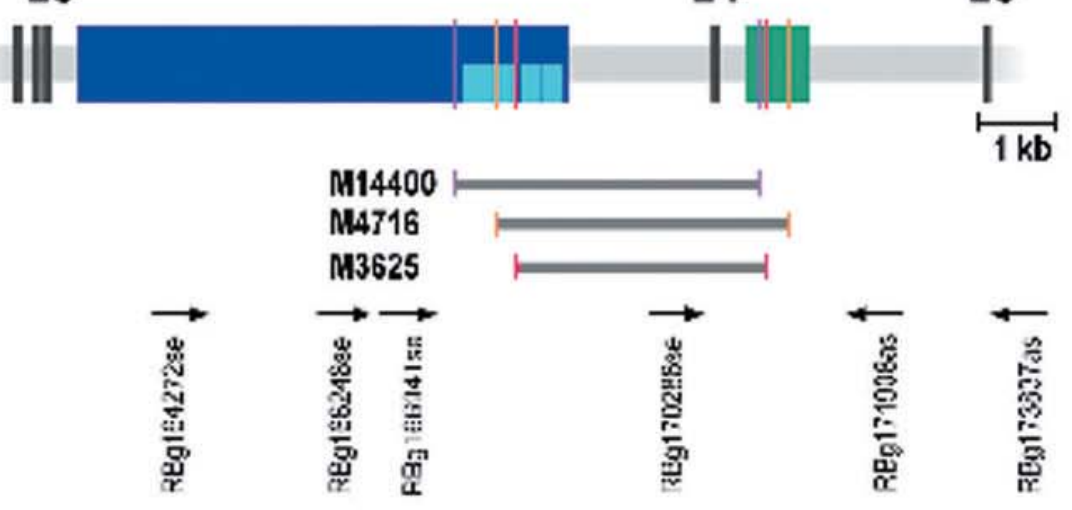

«

B

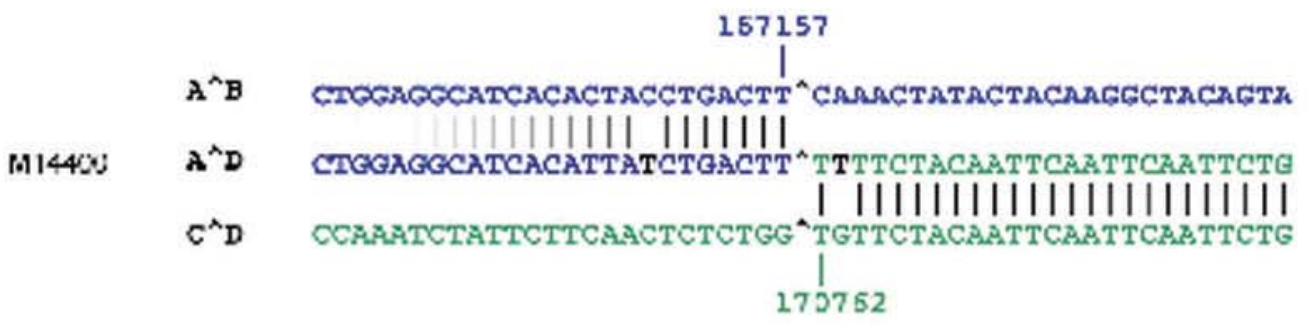

167672

।

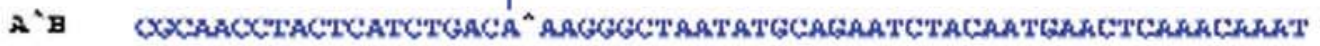

$M<716$

$A^{\wedge} \mathrm{D}$

CGCAACCTACTCATCTOACA^TCATACTGAGTATGATACT^TZGGCATZZTTGRTTZACTC

$c^{*} \mathrm{H}$

||||||||||||||||||

AACCTTATTGTGAGGGGTTTTTATGGGGGTTTCATTATA^TZGGCZTA.ATGATTAZCTC

171215

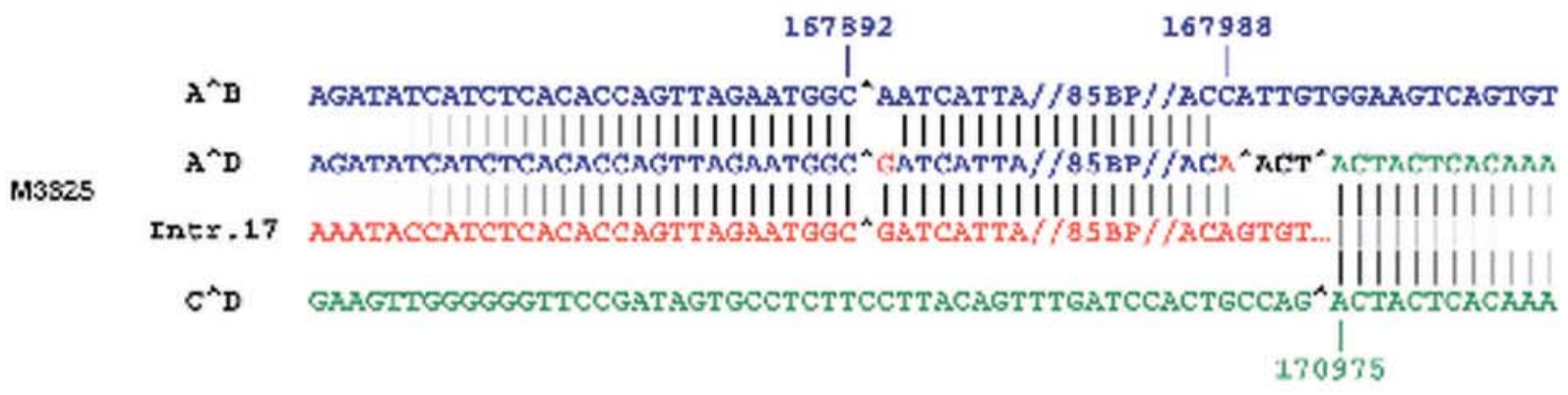

Figure 3 (A) Genomic organisation of a part of the RB1 gene and location of repetitive elements L1PA5, L1HS, and MER21B. The locations of exon 17 and exons 21 to 25 are indicated by vertical grey bars. Sequence similarity between L1HS and L1PA5 is indicated by light and dark blue colour. The 95 bp region identical to the sequence at the 5' breakpoint of patient M3625 is indicated by a vertical red bar in intron 17. (B) Nucleotide sequence alignment of the deletion mutants $\left(A^{\wedge} D\right)$ and the normal allele. Normal sequences from the $5^{\prime}$ breakpoints $\left(A^{\wedge} B\right)$ within the L1HS are displayed in blue, normal sequences from the $3^{\prime}$ breakpoints $\left(C^{\wedge} D\right)$ within the MER21B in green, and the sequence from intron 17 in red. 
A

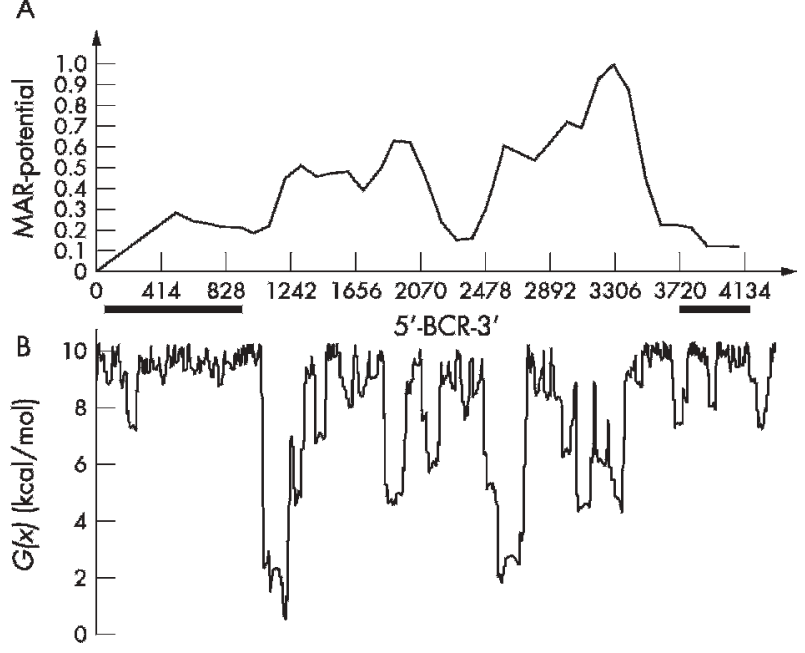

Figure 4 MAR potential of the $B C R$ regions (indicated by the black bars) according to two algorithms, Mar-Finder (A) and SIDD (B). A S/ MAR is a base-unpairing region that is characterised by a succession of destabilised sites, the so called unpairing elements. The flanks of a S/ MAR give rise to DNA transition structures which in turn are the preferred targets of retroelement integration, deletion, and translocation events as described in the text. Such events occur neither in the centre of a S/MAR nor in extended S/MAR free regions. ${ }^{16} 19$

several sequence changes that truncate the two open reading frames that are part of a functional L1. ORF1 and ORF2 of an Ll element encode an RNA binding protein and a protein with endonuclease and reverse transcriptase activities, respectively. Both proteins are necessary for the retrotransposition activity of these autonomous transposable elements. ${ }^{4}$ As sequence changes have destroyed the coding potential of the LlHS in intron 23 of the RBI gene, this element is most likely not an active LINE. Therefore, a deletional mechanism involving active retrotransposition of this transposon is unlikely. The $3^{\prime}$ breakpoints of the three deletions have a maximum distance of only $235 \mathrm{bp}$ and are located in a MER21B DNA transposon fossil. ${ }^{13}$ LIHS and MER21B belong to different classes of interspersed repetitive DNA and show no significant sequence similarity. A search for direct and reverted repeats as well as symmetric elements (using DNASTAR software) identified no significant sequence similarity between $5^{\prime}$ and 3' breakpoint regions. Therefore, the most common pattern for the occurrence of deletions including the formation of deletion facilitating secondary structures due to sequence similarities between proximal and distal breakpoint areas, as described by Chuzhanova et al, ${ }^{2}$ is not a likely explanation for the deletions observed in this work.

In the mammalian nucleus the genome is subdivided into about 60000 chromatin loops by periodic attachments to a nuclear substructure called the nuclear matrix or nuclear scaffold. ${ }^{14-19}$ These attachment points are provided by S/MARs (scaffold/matrix attachment regions), that is, sequences of a minimum length of $300 \mathrm{bp}$ without an apparent sequence consensus. ${ }^{15}{ }^{17}$ Although prototype elements are AT rich, a distinct distribution of AT rich tracts rather than overall base composition determines their activity. ${ }^{18}$ This pattern mediates a propensity for local strand unpairing ${ }^{14}{ }^{15}$ which is, at least in part, utilised in the living cell. Owing to this base-unpairing potential, S/MARs have been considered as recombinogenic structures ${ }^{16}$ and therefore as the preferred sites for integrating retroelements. ${ }^{20}$ Retroelements have consequently been interpreted as markers for the presence of a S/MAR. ${ }^{16}$
While these facts have been elaborated some time ago, more thorough recent analyses have shown that sites of genomic fragility mainly arise at the edges of a S/MAR, that is, at transition structures between B-type and ss-DNA which are frequently characterised by increased accessibility for DNAse. ${ }^{16}{ }^{19}$ The matrix binding sequence itself is largely protected from recombination events, probably because they are associated with proteins of ss-DNA recognition potential. Our present study suggests that also in case of the $R B I$ gene S/MARs flanking regions are the preferred sites triggering deletion events.

During recent years, several software tools have been developed for the prediction of S/MARs. An early example, the "MAR-finder"21 (now known as MARwiz, http://www. futuresoft.org/MarFinder/) is based on the statistical occurrence of S/MAR motifs typical for replication origins, TG rich motifs, curved/kinked DNA, topoisomerase II consensuses, and AT rich sequences. The "stress induced duplex destabilisation" (SIDD) approach identifies regions of DNA unwinding associated with nuclear matrix binding using a statistical mechanical procedure. ${ }^{15} 20$ There are many cases where the prediction of these two approaches is in close agreement. ${ }^{20}$ Figure 4 shows a superimposition of both analysis schemes. Here the MAR-finder positions the two breakpoint cluster region (BCR) genes in locations of minimum MAR potential (0.1-0.3). In the case of the SIDD profile, the centre (approximate map positions 1100-3500) is marked by a regular succession of destabilised sites which are analysed at a standard superhelical density of $\sigma=-0.055$. This architecture is typical of strong S/MAR elements as it allows the mass binding mode for prototypical matrix proteins such as the lamins and scaffold attachment factor $A .{ }^{17}{ }^{18}$ On both sides of this base-unpairing region there are segments of DNA that are stabilised at about $8-10 \mathrm{kcal} / \mathrm{mol}$ and, based on the combined evidence of both analysis schemes, do not have a S/ MAR character. It is clearly seen that these S/MAR associated regions are the primary locations of the deletion endpoints thus confirming the concept that emerged from previous studies.

In conclusion, we have identified two deletion breakpoint clusters in the $R B I$ gene that are involved in recurrent gross deletions in patients with retinoblastoma. The putative mechanism underlying these mutations highlights the importance of S/MARs for deletion formation in humans.

\section{ELECTRONIC-DATABASE INFORMATION}

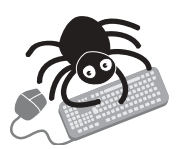

The URL of the Human Gene Mutation Database is www.hgmd.org and that of the MAR-finder is http:// www.futuresoft.org/MarFinder/.

\section{Authors' affiliation}

P Albrecht, K Buiting, D R Lohmann, Institut für Humangenetik, Universitätsklinikum Essen, Essen, Germany

J Bode, GBF, German Research Center for Biotechnology/Epigenetic Regulation, D-38124 Braunschweig, Germany

A K Prashanth, UC Davis Genome Center Davis, Davis, CA 956168816, USA

This study was supported by the Deutsche Forschungsgemeinschaft (Klinische Forschergruppe Ophthalmologische Onkologie und Genetik, DFG KFO 109/1-1, TP II-1).

Conflict of interest: none declared.

Ethics approval: Diagnostic mutation analysis was performed conforming to national guidelines. Scientific analysis of the data was approved by the ethics committee of the Medizinische Fakultät der Universität Essen (file \# 01-100-1700). 
Correspondence to: Dietmar Lohmann, Institut für Humangenetik, Universitätsklinikum Essen, Hufelandstrasse 55, IGI, 45122 Essen, Germany; retinoblastoma@genetik-nrw.de

Revised version received 30 June 2004

Accepted for publication 2 July 2004

\section{REFERENCES}

1 Stenson PD, Ball EV, MortM, Phillips AD, Shiel JA, Thomas NS, Abeysinghe S, Krawczak M, Cooper DN. Human Gene Mutation Database (HGMD): 2003 update. Hum Mutat 2003;21(6):577-81.

2 Chuzhanova N, Abeysinghe SS, Krawczak M, Cooper DN. Translocation and gross deletion breakpoints in human inherited disease and cancer II: potentia involvement of repetitive sequence elements in secondary structure formation between DNA ends. Hum Mutat 2003;22(3):245-51.

3 Richter S, Vandezande K, Chen N, Zhang K, Sutherland J, Anderson J, Han L, Panton R, Branco P, Gallie B. Sensitive and efficient detection of RBI gene mutations enhances care for families with retinoblastoma. Am J Hum Genet 2003;72(2):253-69.

4 Ostertag EM, Kazazian HH Jr. Biology of mammalian L1 retrotransposons. Annu Rev Genet 2001;35:501-38.

5 Kunkel LM, Smith KD, Boyer SH, Borgaonkar DS, Watchel SS, Miller OJ Breg WR, Jones HW Jr, Ray JM. Analysis of human Y-chromosome specific reiterated DNA in chromosome variants. Proc Natl Acad Sci U S A $1977 ; 74(3): 1245-9$.

6 Lohmann DR, Brandt B, Hopping W, Passarge E, Horsthemke B. The spectrum of RB1 germ-line mutations in hereditary retinoblastoma. Am J Hum Genet 1996:58(5):940-9.

7 Lohmann DR, Gerick M, Brandt B, Oelschlager U, Lorenz B, Passarge E, Horsthemke B. Constitutional RB1-gene mutations in patients with isolated unilateral retinoblastoma. Am J Hum Genet 1997;61(2):282-94.

8 Zeschnigk M, Lohmann D, Horsthemke B. A PCR test for the detection of hypermethylated alleles at the retinoblastoma locus. J Med Genet 1999;36(10):793-4.

9 Bremner R, Du DC, Connolly-Wilson MJ, Bridge P, Ahmad KF, Mostachfi H, Rushlow D, Dunn JM, Gallie BL. Deletion of RB exons 24 and 25 causes lowpenetrance retinoblastoma. Am J Hum Genet 1997;61(3):556-70.
10 Houdayer C, Gauthier-Villars M, Lauge A, Pages-Berhouet S, Dehainault C, Caux-Moncoutier V, Karczynski P, Tosi M, Doz F, Desjardins L, Couturier J, Stoppa-Lyonnet D. Comprehensive screening for constitutional RB1 mutations by DHPLC and QMPSF. Hum Mutat 2004;23(2): 193-202.

11 Lohmann DR. RB1 gene mutations in retinoblastoma. Hum Mutat 1999; 14(4):283-8

12 Toguchida J, McGee TL, Paterson JC, Eagle JR, Tucker S, Yandell DW Dryja TP. Complete genomic sequence of the human retinoblastoma susceptibility gene. Genomics 1993;17(3):535-43.

13 The International Human Genome Sequencing Consortium. Initial sequencing and analysis of the human genome. Nature 2001;409(6822):860-921.

14 Bode J, Kohwi Y, Dickinson L, Joh T, Klehr D, Mielke C, Kohwi-Shigematsu T. Biological significance of unwinding capability of nuclear matrix-associating DNAs. Science 1992;255(5041):195-7.

15 Benham C, Kohwi-Shigematsu T, Bode J. Stress-induced duplex DNA destabilization in scaffold/matrix attachment regions. J Mol Biol 1997; 274(2): 181-96.

16 Bode J, Benham C, Ernst E, Knopp A, Marschalek R, Strick R, Strissel P. Fatal connections: when DNA ends meet on the nuclear matrix. J Cell Biochem Suppl 2000;35:3-22

17 Bode J, Benham C, Knopp A, Mielke C. Transcriptional augmentation: modulation of gene expression by scaffold/matrix-attached regions (S/MAR elements). Crit Rev Eukaryot Gene Expr 2000;10(1):73-90.

18 Bode J, Goetze S, Heng H, Krawetz SA, Benham C. From DNA structure to gene expression: mediators of nuclear compartmentalization and dynamics. Chromosome Res 2003;11(5):435-45.

19 Hensel JP, Gillert E, Fey GH, Marschalek R. Breakpoints of $t(4 ; 11)$ translocations in the human MLL and AF4 genes in ALL patients are preferentially clustered outside of high-affinity matrix attachment regions. J Cell Biochem 2001;82(2):299-309.

20 Goetze S, Huesemann Y, Baer A, Bode J. Functional characterization of transgene integration patterns by halo fluorescence in situ hybridization: electroporation versus retroviral infection. Biochemistry 2003;42(23):7035-43.

21 Singh GB, Kramer JA, Krawetz SA. Mathematical model to predict regions of chromatin attachment to the nuclear matrix. Nucleic Acids Res 1997;25(7):1419-25. 